\title{
Correction to: Towards a stochastic programming modeling framework for districting
}

\author{
Antonio Diglio ${ }^{1}$ (D) $\cdot$ Stefan Nickel ${ }^{2,3}$ (D) $\cdot$ Francisco Saldanha-da-Gama ${ }^{4,5}$ (D)
}

(c) Springer Science+Business Media, LLC, part of Springer Nature 2020

\section{Correction to: Annals of Operations Research https://doi.org/10.1007/s10479-020-03631-7}

This corrections has been published because several proofing corrections were overlooked during proofing.

Third authors' name should appear as: Francisco Saldanha-da-Gama

Author ORCID to be read as:

Stefan Nickel:

orcid.org/0000-0002-8339-0117 <http://orcid.org/0000-0002-8339-0117>

Francisco Saldanha-da-Gama:

orcid.org/0000-0002-2074-1856 <http://orcid.org/0000-0002-2074-1856>

Original article has been updated thus.

Publisher's Note Springer Nature remains neutral with regard to jurisdictional claims in published maps and institutional affiliations.

The original article can be found online at https://doi.org/10.1007/s10479-020-03631-7.

Antonio Diglio

antonio.diglio@unina.it

1 Department of Industrial Engineering (DII), Università degli Studi di Napoli Federico II, Piazzale Tecchio, 80, 80125 Naples, Italy

2 Institute of Operations Research, Karlsruhe Institute of Technology (KIT), Karlsruhe, Germany

3 Department of Logistics and Supply Chain Optimization, Research Center for Information Technology (FZI), Karlsruhe, Germany

4 Dept. Estatística e Investigação Operacional, Faculdade de Ciências da Universidade de Lisboa, 1749-016 Lisbon, Portugal

5 Centro de Matemática, Aplicações Fundamentais e Investigação Operacional, Faculdade de Ciências da Universidade de Lisboa, 1749-016 Lisbon, Portugal 